\title{
Potencial y Problemas de la Simulación en Ambientes Virtuales para el Aprendizaje
}

\author{
Claudio A. Fredes ${ }^{(1)}$, Juan P. Hernández ${ }^{(1)}$ y Daniel A. Díaz ${ }^{(2)}$ \\ (1) Universidad Católica del Maule, Facultad de Ciencias Agrarias y Forestales, \\ Escuela de Agronomía, Camino a los Niches Km. 6, Curicó-Chile (e-mail: cfredes@ucm.cl; \\ jhernandez@ucm.cl) \\ (2) Pontificia Universidad Católica de Chile \& EBM Consulting, Av. Apoquindo 5106, Of. 21, \\ Las Condes, Santiago-Chile (e-mail: danieldiaz@uc.cl)
}

Recibido Sep. 01, 2011; Aceptado Oct. 06, 2011; Versión final recibida Nov. 04, 2011

\begin{abstract}
Resumen
En este artículo se presenta información que busca establecer una base sobre la cual discutir el desarrollo de un software de enseñanza basado en simulación de ambientes virtuales, para su uso por parte de administradores y estudiantes del área técnica de manufactura en Chile. El objetivo general fue revisar la potencialidad y problemas de uso de software de simulación en nuestra sociedad digital. Se estudia críticamente el uso real de las Tecnologías de la Información y Comunicación (TIC) en la educación actual y se analiza la problemática en el uso pedagógico de los ambientes virtuales. Se concluye que el uso de las TIC debe tomar en consideración que las instituciones educativas han de desarrollar estrategias de apoyo al docente para que el uso de las TIC sea realmente un elemento positivo en el proceso formativo de los alumnos.
\end{abstract}

Palabras clave: cibercultura, TIC, software de simulación, ambientes virtuales

\section{Potential and Problems of Simulation in Virtual Learning Environments}

\begin{abstract}
This article provides information to establish a basis for discussing the development of teaching software based on virtual environments, to be used by administrators and students of the technical area of manufacture in Chile. The main objective was to analyze the potentiality and the problems that the use of simulation software implies in our digital society. The real use of Information and Communication Technologies (ICT) in the present educational system is critically discussed and the problem of the use of virtual environments in teaching is analyzed. It is concluded that the use of ICT must consider that educational institutions in Chile must develop supporting strategies for the teachers so the use of ICT really represents a positive element in the formation process of the students.
\end{abstract}

Keywords: cyberculture, ICT, simulation software, virtual environments 


\section{INTRODUCCIÓN}

En la cibercultura, las Tecnologías de la Información y la Comunicación (TIC) tienen un rol clave para mejorar las destrezas cognitivas, motivar el análisis cooperativo de problemas y contribuir con la labor docente (Díaz-Antón, 2002; López, 2011). Específicamente, los software educativos son las herramientas educativas en la nueva sociedad del aprendizaje (Bustos y Coll, 2010). Sin embargo, su potencial pedagógico está siendo desaprovechado por los profesores (Proto, 2010), sobretodo en países subdesarrollados donde, la llamada brecha digital segrega a una parte importante de los alumnos y dificulta el pleno uso de este tipo de herramientas tecnológicas (Serrano y Martínez, 2003; Quinche y González, 2011).

El uso extensivo de TIC ha tenido un impacto creciente en las instituciones de educación superior, con impactos no solo hacia el interior de la institución, sino también en su vinculación para el trabajo conjunto con otras instituciones (UNESCO, 2009). No obstante, su uso podría ser inconsistente y variable entre cursos e instituciones analizando causas institucionales y estructurales, atribuyendo responsabilidades a docentes, administrativos y proveedores de TIC (Selwin, 2007). Adicionalmente, diversos autores señalan que la simple inserción de las TIC en instituciones educativas, no garantiza su eficacia (Kreinjs et al., 2003; Proto, 2010; Hernández y Romero, 2010; Fernández, 2005).

Cuando se han adoptado estrategias a nivel institucional y de aula para que los alumnos usen adecuadamente software educativos; la simulación en ambientes virtuales puede ser una poderosa herramienta para situar a los estudiantes en escenarios "prácticos" a los que difícilmente podrían acceder en la realidad. Existen, en este sentido, dos categorías de software que recrean ambientes virtuales y que pueden usarse en el aprendizaje: los simuladores y los videojuegos. Dentro de los simuladores, hay un grupo de restringido acceso, que precisan de hardware especializados para simular acciones físicas y otro grupo, que usando los hardware comunes, sitúan al usuario en escenarios virtuales para aprender a realizar tareas "prácticas" (Pan et al., 2006). Por otro lado los videojuegos en primera persona, poseen tres características que pueden potenciar el aprendizaje en los Ambientes Virtuales (AV) donde se desarrollan: a) un objetivo claramente definido, que es necesario alcanzar para ganar el juego; b) una serie de obstáculos (mientras más obstáculos más desafiante es el juego, pocos obstáculos lo tornan aburrido), que son necesarios superar para alcanzar el objetivo; y c) la colaboración o la competencia con o contra otros jugadores (sean estos reales o virtuales) en orden a alcanzar el objetivo (Smith Robbins, 2011).

En este artículo se presenta información que busca establecer una base sobre la cual discutir el desarrollo de un software instruccional, basado en simulación de AV, para su uso por parte de trabajadores, certificadores de competencias y estudiantes del área técnica de manufactura en Chile. El objetivo general fue revisar la potencialidad y problemas de uso de software de simulación en nuestra sociedad digital. Específicamente, pretendimos estudiar críticamente el uso real de las TIC en la educación actual y analizar la problemática en el uso pedagógico de los AV. Para abordar el problema de investigación del presente artículo, fue necesario desarrollar una aproximación a los diversos elementos relacionados con herramientas de instrucción basadas en AV. La búsqueda de información consideró los motores de búsqueda científica "Science Direct" (Elsevier), CAB Abstract, ISI Thompon Reuters y el motor general de búsqueda académica "Google Scholar". Los antecedentes presentan las características que ha adoptado la educación en la cibercultura, la condición de los estudiantes en este contexto y la situación de los profesores. Luego se presenta una discusión que recoge las limitaciones actuales de uso de TIC en los alumnos, las posibilidades que entregan los AV y el rol de los profesores en este escenario emergente.

\section{LA CIBERCULTURA}

En la era de la digitalización, la tecnología informática ha ido creciendo exponencialmente, aparece la "red" como el nuevo medio de comunicación con interconexión mundial con cientos de millones de usuarios (Lévy, 2007), perfilando una nueva cultura que replantea cómo relacionarnos, 
es así que ahora la distancia no es una limitante para comunicarnos (Escobar, 2005). Este es el escenario de la "cibercultura", definida como el conjunto de técnicas, actitudes, valores y modos de pensamiento que confluyen en el ciberespacio. El término cibercultura fue acuñado por Pierre Lévy (op.cit.). El citado libro analiza cómo la tecnología digital construye nuevos aspectos culturales, reformulándolos desde la cultura actual (Escobar, 2005). Este mismo autor señala que la cibercultura transforma las relaciones sociales y la forma de hacer ciencia, vale decir, una ciencia más electrónica que exige replantearse las bases epistemológicas de ella; de esta forma ha nacido el término e-epistemología para entender mejor cómo generar y adquirir conocimiento en la cibercultura (Vallverdú, 2008). En esta sociedad, las tecnologías digitales aparecen como las formas dominantes para comunicarse, compartir conocimiento, investigar, producir, organizarse y administrar. Tecnología, cultura y sociedad están íntimamente relacionadas en la cibercultura. Es así como las nuevas generaciones de estudiantes gastan más de 12 veces de su tiempo frente a un aparato tecnológico que leyendo un libro (Beastall, 2006), de tal modo que, según sugiere Gros (2006), la escuela debería formar parte de la sociedad informacional y debería ser el espacio social en que se re-construye y se interpreta la cultura mediática.

\section{La educación en la cibercultura}

En la cibercultura, la educación utiliza las tecnologías digitales para generar una mejor gestión del conocimiento, quienes poseen plataformas tecnológicas tienen mejor acceso económico, cultural y social, accediendo a trabajos "inteligentes". Por otro lado, quienes carecen de información se sitúan a gran brecha de distancia, poseen trabajos de baja productividad y salario, están privados del diálogo y de gran parte del intercambio cultural, por ende, tienen mucho menos oportunidades de movilidad social (Hopenhayn, 2003). Esta brecha digital es la segmentación y distanciamiento entre aquellos que tienen y utilizan las TIC y aquellos que no saben usarlas o no las poseen. Superar esta brecha es un importante eje de las políticas públicas de países subdesarrollados y en vías de desarrollo. Se ha visto que ello no es sólo la falta de acceso a Internet e infraestructura computacional, sino que también el déficit socioeconómico y cultural para usar adecuadamente estas tecnologías (Serrano y Martínez, 2003).

En términos de las características generales de este tipo de herramientas, se señala que los software educativos deben ser flexibles y modulares para diversos usuarios, colaborativos, estimular la comunicación interpersonal y la evaluación grupal (Calzadilla, 2001), permitir la autoevaluación formativa y generar feedback inmediato para evaluación autónoma (Cebrián, 2003). Físicamente, la nueva aula que piden las TIC debe ser diferente y estar descontextualizada del entorno clásico (Adell, 1997) y en cuanto a su ambiente Hernández y Romero (2010) recomiendan un entorno de aprendizaje abierto para generar una verdadera comunidad de aprendizaje. Tecnologías de información para la educación como los videojuegos y simuladores de escenarios, pueden servir para transmitir contenidos complejos o de poco interés para los jóvenes, potenciar la motivación y el interés de los alumnos. Específicamente, los videojuegos atraen a los estudiantes con representaciones multi-sensoriales compuestas de imágenes, sonidos y modalidades kinectésicas, ayudan a enfrentar frustraciones, fomentar la sociabilidad, posibilitar la reflexión, el discurso y el análisis colectivo en el aula (Rosas et al., 2003).

\section{Estudiantes y su relación con la realidad virtual}

En educación, los alumnos experimentan a través de manipulaciones físicas en tiempo real (Pan et al., 2006) y la Realidad Virtual (RV) es la interacción multisensorial e intuitiva y la inmersión en un ambiente virtual. Helsel (1992) describe los ambientes virtuales como un espacio que permite a los usuarios convertirse en participantes de un mundo abstracto, donde la posición física del objeto no existe. La RV puede ser inmersiva, definida como aquellos sistemas donde el usuario se siente dentro del mundo virtual que está explorando por causa de diferentes dispositivos denominados accesorios; RV semi-inmersiva, que se caracteriza por disponer de cuatro pantallas en forma de cubo (tres pantallas forman las paredes y una el piso), las cuales rodean al observador, el usuario usa lentes y existe un dispositivo de seguimiento de movimientos de la cabeza; finalmente los sistemas no inmersivos o de escritorio, son aquellos donde el monitor es la ventana hacia el mundo virtual y la interacción es por medio del teclado, micrófono, mouse o 
joystick. Otra categorización de la RV corresponde a la de Cronin (2000), quien las organiza por el grado de inmersión - concepto que se trabajará mas adelante - que el sistema provee: no inmersiva, en ella las imágenes se observan por una pantalla o monitor, aunque sean imágenes en tres dimensiones, es la mas común y menos costosa de la RV. Semi inmersiva; donde las imágenes y los efectos son proyectados en una pantalla, la inmersión es alta pero la interacción se ve afectada. Totalmente Inmersiva: esta es la más costosa de las RV, requiere de interfases especiales como guantes electrónicos, pantallas estereoscópicas, trajes virtuales, rampas, sistemas de audio de alta fidelidad, cascos, entre otros.

Pantelidis (1993) destaca que en la RV se generaría aprendizaje debido a la participación activa y protagonista del alumno en un sistema de alta individualización que se adapta a su ritmo de aprendizaje. Estos sistemas educativos, basados en la experiencia en primera persona y en la interacción con otros, son modelos basados en el constructivismo (Bricken, 1990). Winn y Windschitl (2000) reportan que el sentido de presencia en primera persona en los ambientes virtuales genera aprendizajes, porque ampliaría las formas de adquirir conocimiento en la interacción. Otras ventajas serían promover la colaboración y ayudar al profesor a ser facilitador de un aprendizaje autónomo de los alumnos (Hamada, 2008). Winn (1993) ha propuesto "tamaño", "transducción" y "reificación" como las características pedagógicas de los entornos basados en RV; tamaño porque los usuarios del AV pueden cambiar su estructura física, pudiendo interactuar en mundos macro y micro, tal como dentro de un átomo (Kontogeorgiou et al., 2008), en una célula o en el sistema solar (Bakas y Mikropoulos, 2003); transducción referido a la capacidad para apropiarse una información que supera el rango de las experiencias posibles del mundo real; reificación porque implica la transformación de ideas abstractas en representaciones perceptibles a través de objetos virtuales.

\section{AMBIENTES VIRTUALES}

Los Ambientes Virtuales son mundos autónomos comunicados que interactúan con un usuario que está situado simultáneamente dentro del computador como fuera de éste. Aparece una importante cualidad psicológica: la presencia, descrita como "la sensación de estar realmente allí", la cual juega un rol clave en los AV (Mikropoulos y Strouboulis, 2004). Según Traphagan et al. (2010) hay tres tipos de presencia; social, cognitiva y de enseñanza. La presencia social es la forma en que los estudiantes se enfrentan social y emocionalmente a una comunidad de aprendizaje virtual. La presencia cognitiva, en cambio, es la forma de construcción de su conocimiento, mediante procesos de reflexión y comunicación. La presencia de enseñanza, finalmente, es aquella que facilita directa o indirectamente la interacción social y la simulación en el proceso cognitivo. La presencia habilita la coordinación de actividades para generar aprendizajes en los AV (Rourke et al., 2001).

La interacción social en $\mathrm{AV}$, tanto con otros alumnos como con el profesor, ejerce una marcada influencia sobre el comportamiento de los estudiantes. Hrastinski (2006) y Kreijns et al. (2003) señalan que la interacción social fluye cuando el AV permite interacción específica informal, tal como se da naturalmente en los campus reales. Uno de los desafíos de la educación en línea sería posibilitar la interacción natural estudiante-estudiante en el mundo virtual (Guri-Rosenblit, 1999; Palloff y Pratt, 1999). La comunicación en AV respecto a su momento de uso puede ser sincronizada como asincronizada, y respecto a la cantidad de usuarios puede ser de un solo usuario o multiusuario. La comunicación sincronizada permite los comentarios inmediatos y hace que los estudiantes se sientan más a gusto de participar que como individuos aislados. Sin embargo, la comunicación asincronizada proporciona a los estudiantes un mayor control y flexibilidad durante el proceso formativo (Hrastinski, 2007). Los AV multi-usuarios poseen ventajas respecto a los de un solo usuario por la colaboración que se produciría (Traphagan et al., 2010). Los mundos virtuales 3D multi-usuarios donde cada usuario tiene su avatar, representación gráfica, tales como Second Life o Virtual Spain han tenido un explosivo crecimiento, como espacios donde compartir experiencias y conocer gente en espacios que poseen una atractiva estética modelada en tercera dimensión. 
La representación de los usuarios puede ser por avatares o agentes. Los primeros son una representación digital perceptible del usuario cuya posibilidad de interactuar dentro del AV está sujeta a las acciones ejecutadas en tiempo real por el ser humano (Galanxhi y Nah, 2007). Los agentes, en cambio, están sujetos a algoritmos computacionales diseñados para cumplir ciertos objetivos (Bailenson y Blascovich, 2004). Los diseñadores de sistemas sociales digitales reconocen la importancia de los avatares como aporte a la sensación de presencia y expresión de identidad de los usuarios. Este aporte puede potenciarse agregando aplicaciones en línea, como juegos de rol, blogs y acceso a diferentes ambientes virtuales (Vasalou y Joinson, 2009).

Existe evidencia videográfica de diversas experiencias educativas que se han desarrollado en AV, como la simulación en ambientes de áreas militares donde se simula una instrucción de disparo; fábricas de automóviles que muestran la línea de proceso de fabricación e inducción en manejo de maquinaria pesada; clínicas y hospitales; fábricas elaboradoras de ácido fosfórico; museos de obras de arte o agroindustrias virtuales. Este último trabajo, del grupo Ingenio Colombiano (IngCo), supuso el desarrollo de un software educativo y de aprendizaje virtual modelado en 3D denominado Virtual Plant. Otros proyectos con mayor tecnología son la simulación de un vuelo espacial de la NASA y el proyecto PRESENCCIA. Este último utiliza la última tecnología de RV disponible para generar un ambiente virtual modelado en 3D controlable mediante impulsos eléctricos cerebrales, el cual contribuiría al desenvolvimiento e inserción de personas discapacitadas en la sociedad.

Ahora bien, cuando en estos ambientes virtuales se agrega la capacidad de simular, el resultado sobre el aprendizaje se incrementa. Se entiende la simulación como el proceso de diseñar una abstracción de un sistema real para poder experimentar y explicar un objeto, idea o sistema mediante un modelo, que es una simplificación de un sistema con entradas (atributos para ajustar el modelo) y salidas (comportamiento de las variables), que dependen de la observación de los fenómenos de éste sistema. Mientras más fenómenos se representen en el sistema, más preciso será el modelo. Los modelos de simulación computacional se pueden clasificar en estocásticos o deterministas, en función del comportamiento del sistema; en estáticos o dinámicos en función del tiempo; y en continuos o discretos según su permanencia. La simulación en AV son modelos dinámicos y deterministas.

Lo más avanzado en simulación en $\mathrm{AV}$ son aquellos con dispositivos o hardware de interfase extras al ambiente virtual. Estos dispositivos externos proporcionan un especial efecto de inmersión en un ambiente interactivo tridimensional (Pan et al., 2006). La industria de video juegos ha llevado esta tecnologías a los hogares. Es el caso de la consola de videojuegos Xbox KINECT ${ }^{\circledR}$ de la compañía Microsoft. Otros modelos de RV logran incluir elementos del mundo real en un $\mathrm{AV}$, lo que se conoce como virtualidad aumentada.

\section{Profesores y sus problemas con las TIC}

El valor social de la incorporación de las TIC en educación se revela cuando genera capacidad de resolver problemas de la vida cotidiana (Fundación Omar Dengo, 2006) y el facilitador de esta capacidad es, sin duda, el profesor. Entonces el buen uso educativo de las TIC pasa por los docentes. En la cibercultura, según Prensky (2001) los alumnos son nativos digitales y los profesores inmigrantes digitales. Así, el inmigrante debe gastar mucho tiempo para ambientarse en su nueva patria. Y muchos profesores no se han dado este tiempo. Es necesario su aporte porque, como señala Hernández y Romero (2010), la simple inserción de las TIC en el aula no garantiza su eficacia educativa. La real introducción pasa por una alfabetización digital de los profesores y por tener una adecuada infraestructura tecnológica (Proto, 2010).

Para asegurar que se inserten claramente las TIC en los contenidos curriculares y en la práctica docente diaria, deben plantearse estrategias de introducción de herramientas digitales apropiadas a la institución educativa (Hernández y Romero, 2010). Los profesores deben entender que estas herramientas digitales se adaptan a un aprendizaje constructivista basado en la solución de problemas (Sosa et al., 2005). Para la construcción de aprendizajes significativos y socio funcionales de los alumnos, se requiere de profesores mediadores y guías de la actividad de sus 
educandos, de esta forma se fomentará de manera física, simbólica y social, la creación de entornos de aprendizaje y la interacción con otros. Un estudio señala que existe una buena alfabetización digital de los profesores chilenos, aunque su uso no incluye efectivamente a los últimos destinatarios, los alumnos y por ende, se desperdicia la real oportunidad de usar las TIC en el aula (MINEDUC, 2009).

Las TIC, como motor de formación de personas en la cibercultura requieren cumplir con todos los requisitos de usabilidad de cualquier software de calidad y, una vez cumplido ello, ser integrado en todos los niveles de un país. Muchos profesores no lo usan para sus clases, o lo hacen deficientemente porque se ven enfrentados a estos problemas de usabilidad y acceso. Las causas que explican ello son una renuencia a desembolsar altos costos en tecnologías que rápidamente quedan obsoletas; enfoque en aprendizajes superficiales anclados en la pura información y analfabetismo digital del cuerpo docente incapaz de conducir los procesos de enseñanza, entre otros (Marquès 2008). Cuando el profesor no guía adecuadamente a sus alumnos, las TIC pueden generar distracciones en los alumnos que se ven sujetos a fuentes de información poco fiables. Incluso se podría generar un trastorno adictivo debido a su uso excesivo, especialmente en adolescentes hombres y con algún trastorno de personalidad y autoestima (Grüsser, 2007), podrían provocar además problemas de sociabilidad al desarrollar en forma individual y aislada su proceso de aprendizaje (Fernández, 2005).

\section{DISCUSIÓN}

De los diversos temas relacionados con las Tecnologías de la Información y Comunicación se discute en lo que sigue algunos aspectos sobre sus limitaciones y sobre el papel que deben jugar los profesores en su utilización.

\section{Limitaciones actuales para un uso activo de las TIC}

La transformación de la educación (CEPAL/UNESCO, 1992) cumple un rol fundamental en las sociedades humanas, al ser el motor de la generación del conocimiento, toda vez que se inserte de manera efectiva en la nueva sociedad del aprendizaje (Bustos y Coll, 2010) y en la cibercultura definida para estos tiempos. Herramientas basadas en las TIC abarcan dicho desafío, al promover el uso de tecnologías vinculadas al proceso formativo. Sin embargo, estas herramientas tecnológicas deben insertarse de manera efectiva dentro del aula, lo que implica una alfabetización de los docentes y alumnos, sumado a estrategias pedagógicas y organizativas apropiadas (Lévy, 2007; Proto, 2010; Hernández y Romero, 2010).

Este tipo de recursos tecnológicos, necesarios para el aprendizaje en el contexto actual, puede estar siendo desaprovechado por parte de docentes y alumnos. Existiría, así, un alto grado de desigualdad educativa, debido principalmente a la escasa disponibilidad de equipos informáticos en los establecimientos y a su uso básico o nulo, desaprovechándose la oportunidad de usar las TIC para mejorar en los estudiantes las destrezas cognitivas, facilitar el aprendizaje por descubrimiento, promover la resolución de problemas y contribuir en los procesos de construcción del conocimiento. La realidad es que los alumnos tienen mayor acceso a Internet y computadores fuera del colegio, por lo que extender el proceso formativo fuera de la sala de clases es la alternativa para los estudiantes de menores recursos (Díaz-Anton, 2002; Serrano y Martínez, 2003; Proto, 2010).

\section{La propuesta de ambientes virtuales para el aprendizaje}

Los AV son un atractivo tipo de software para fortalecer el aprendizaje, su uso ha ido pasando desde usuarios de elite (medicina, biotecnología, e ingeniería compleja) hasta usuarios comunes (educación básica y prebásica), en estos últimos años. Su uso plantea una serie de ventajas y una oportunidad para superar las brechas tecnológicas en educación. El constructivismo, modelo pedagógico implícito en los AVA, estimula la adquisición de conocimientos por descubrimiento, esto es especialmente importante en sectores educacionales con menores recursos y con difícil 
acceso a equipamientos que podrían ser simulados en AV (Serrano y Martínez, 2003; Dalgarno et al., 2009; Cheng y Wang, 2011).

Los AV, basados en tecnologías de Realidad Virtual (RV), pueden ser utilizados en los procesos de aprendizaje debido a una serie de características relacionadas con aspectos de interacción con el usuario, proveyendo espacios interactivos modelados en tres dimensiones (3D), los cuales confluyen en el concepto de presencia, factor fundamental para la propuesta educativa de los AV (Mikropoulos y Strouboulis, 2004; Pan et al., 2006); a medida que aumenta la sensación de presencia en el mundo virtual, se incrementa el rendimiento de los alumnos y consecuentemente mejora el proceso de aprendizaje. Criterios de realismo y, consecuentemente, control del entorno, favorecen la sensación de presencia (De Lucia et al., 2009). La interacción social juega un rol clave en los procesos de enseñanza mediados por computador, aunque su incorporación en los AV no garantiza por si solo buenos resultados en el aprendizaje. No obstante, se ha visto que la presencia de un tutor virtual fortalece el aprendizaje de sus alumnos, influyendo en un aspecto anímico también, dado por la posibilidad de interactuar con sus compañeros (Galvis, 1992; De Lucia et al., 2009).

Las características que designan a la RV como una prometedora y potente herramienta educacional aún no están claras (Mikropoulos y Natsis, 2011). Como señala Salzman et al. (1999) el aprendizaje es un proceso complejo y las características de los ambientes de aprendizaje no actúan de forma aislada, todas ellas juegan un rol en el proceso y en los resultados. Diversos estudios relacionados a la RV para aplicaciones educativas argumentan acerca de su uso como una herramienta educacional. Aunque no existen aún evaluaciones exactas para los AV, se propone evaluarlos según su propio mérito, aunque siempre basados en los principios del constructivismo (Bricken, 1990; Brown et al., 1998; Mikropoulos y Natsis, 2011).

Por sus ventajas, la RV está popularizándose, a través de software y hardware aplicados en plataformas sociales, para educación y en videojuegos en ambientes virtuales, como también en investigación de punta para situar discapacitados en AV. De este modo, nos acercamos a la descripción de William Gibson (1984), quien en su libro Neuromancer definió el ciberespacio como un sitio donde la gente puede experimentar la realidad virtual, interactuando con computadores; ciencia ficción que se llevó al cine en la película The Matrix (1999) y AVATAR (2009). AV basados en tecnologías de RV tienen un potencial educativo, un ejemplo de estos son los videojuegos, o la "gamification" de la educación, la cual se fundamenta en la simpleza de sus características, que se pueden aplicar a la vida propia del estudiante; para quienes la graduación es el objetivo final, los obstáculos son las dificultades propias del avance de la carrera y la presencia de colaboradores que ayudan y competidores, que dificultan, es propio de las relaciones sociales de todo estudiante en su comunidad educativa (Smith-Robbins, 2011).

La simulación de AV constituye una alternativa viable de mejora de los procesos de enseñanza, toda vez que su inserción en el aula sea acogida en forma satisfactoria por docentes y alumnos. La adopción de técnicas de aprendizaje extendido complementaría dicho proceso tomando en cuenta la mayor accesibilidad de los estudiantes a estas tecnologías fuera de la sala de clases. La implementación de instrumentos tecnológicos basados en RV, contribuye en el perfeccionamiento de los procesos cognitivos de los estudiantes y apoya la labor docente, siempre y cuando su inserción en el aula sea realizada de manera efectiva, lo que implica una reorganización de las políticas educacionales, sumado a una constante capacitación por parte de los usuarios y beneficiarios, vale decir, profesores y alumnos.

\section{Rol del profesor y de las escuelas}

La Octava Encuesta de Actores del Sistema Educativo en Chile (CIDE, 2010), señala que el 30\% de los alumnos le asigna un rol al profesor al establecer el impacto de las TIC en el aprendizaje, visión que contrasta con el $70 \%$ de respuestas positivas por parte de los profesores ante la misma pregunta. Pareciera ser que los profesores se visualizan en un rol que no es apreciado por los alumnos, o que al menos no ven como decisivo para asegurar el aprendizaje mediante estas tecnologías. 
Un desafío central al momento de desarrollar un programa computacional que pretende integrarse a las prácticas educativas, es visualizar que éste no solo debe incorporar al estudiante, sino también al profesor. La función docente de mediación al aprendizaje opera en diversos niveles, y en ese sentido es factible que estas funciones se transfieran al uso de un software (García y Benítez, 2011). Así, al estar el docente capacitado no solo para detectar el error -función que un software puede hacer perfectamente- sino también para comprender la fuente de tal error, contará con más información para iniciar acciones que eviten nuevos errores. Del mismo modo, la mirada sobre las actitudes va más allá que el enfoque en la ejecución, y se detiene en el modo en que se logran los objetivos trazados. Por ejemplo, todas las temáticas de seguridad industrial apuntan no solo a la productividad, sino también a la seguridad personal, a la sustentabilidad del negocio y a la continuidad de la operación, por lo que el enfoque exclusivo en la tarea puede obviar estos tópicos, comunicando en tal caso, y de modo erróneo, que lo único importante es la tarea y los objetivos, independiente del modo en que sean alcanzados.

El desafío pendiente es que los docentes adquieran habilidades de manejo y gestión de este tipo de herramientas. De modo que al momento de recibirlas logren hacerlas calzar con una programación lógica de contenidos, y tales programas sean efectivamente una herramienta instruccional más, que viene a complementar las otras que pueda tener disponible en su oferta didáctica. En la medida que los docentes no planifiquen sus clases, o que no hagan seguimiento de estas actividades, es altamente probable que los logros de aprendizaje no se alcancen en todos los estudiantes, y que sean exitosos solo aquellos que por variables distintas al profesor tengan motivación por el aprendizaje. Los problemas descritos en USA para un adecuado uso de las TIC (Selwin, 2007), son en mayor grado, aplicables a países menos desarrollados como Chile; ¿Estamos preparando a los profesores para que sean facilitadores de sus alumnos en el uso de las TIC? Para un efectivo uso de las TIC, se hace necesario estructuras institucionales que faciliten, profesores preparados y software de calidad y pertinentes, pues la mayor disposición de computadores, no garantiza su eficacia en términos educativos (Kreinjs et al., 2003; Proto, 2010; Hernández y Romero, 2010 y Fernández, 2005).

\section{CONCLUSIONES}

1. El uso de las TIC constituye una oportunidad para poder insertar de manera efectiva y eficiente herramientas tecnológicas en el aula ya que aportan al proceso de transformación de la educación en la cibercultura. Además permiten establecer un mayor grado de equidad en el proceso de enseñanza, aunque deben generarse estrategias educativas y administrativas que sirvan de base para implementarlas, lo que incrementaría las posibilidades para su completo aprovechamiento por parte de los profesores y los estudiantes.

2. Los AV son un atractivo tipo de software para facilitar el proceso formativo, contribuyendo a su vez a superar la brecha digital existente en la educación. Se encontró evidencia respecto a que los $\mathrm{AV}$ basados en tecnología de RV generarían un mayor efecto sobre el aprendizaje al poseer características de interacción y presencia, factores fundamentales en el proceso de enseñanza y aprendizaje.

3. En el desarrollo de un software educativo se debe contemplar las características e intereses de los estudiantes, como también los conocimientos y competencias de los profesores. Se deberá fijar objetivos en forma integradora y visualizar los problemas de diseño y estructura educativa para que cumpla la función de apoyo a la labor docente y facilite el proceso de enseñanza aprendizaje.

\section{AGRADECIMIENTOS}

Los autores agradecen al programa TIC EDU de FONDEF - Fondo de Fomento al Desarrollo Científico y Tecnológico del Gobierno de Chile por financiar esta publicación, que se enmarca en el proyecto "Vinificación Virtual: desarrollando competencias para el manejo de bodegas de vino" TE10I009. 


\section{REFERENCIAS}

Adell, J., Tendencias en educación en la sociedad de las tecnologías de la información, Revista electrónica de tecnología educativa (en línea), 1997, http://www.uib.es/depart/gte/edutece/revelec7/revelec7.html. Acceso: 25 de marzo (2011).

Bailenson, J.N. y Blascovich, J., Avatars, In W. S. Bainbridge (Ed.), Encyclopedia of humancomputer interaction (pp. 64 - 68). Great Barrington MA: Berkshire Publishing Group (2004).

Bakas, C. y T.A. Mikropoulos, Design of virtual environments for the comprehension of planetary phenomena based on students' ideas, International Journal of Science Education: 25(8), 949-967 (2003).

Beastall, L., Enchanting a disenchanted child: revolutionising the means of education using Information and Comunication Technology and e-learning, British Journal of Sociology of Education: 27(1), 97-110 (2006).

Bricken, W., Learning in virtual reality, Technical report No. HITL-M-90-5. (en línea), 1990, http://www.hitl.washington.edu/publications/m-90-5/. Acceso: 25 de marzo (2011).

Brown, D. y otros tres autores, Development and evaluation of the virtual city, The international journal of virtual reality: 3(4), 27-38 (1998).

Bustos, A. y C. Coll, Los Entornos Virtuales como Espacios de Enseñanza y Aprendizaje. Una perspectiva psicoeducativa para su caracterización y análisis, Revista Mexicana de Investigación Educativa: 15(44), 163-184 (2010).

Calzadilla, M., Aprendizaje colaborativo y tecnologías de la información y comunicación, Revista Iberoamericana de Educación, línea), 2001, http://www.rieoei.org/deloslectores/322Calzadilla.pdf. Acceso: 24 de marzo (2011).

Cebrián, M., Innovar con tecnologías aplicadas a la docencia universitaria, Madrid (2003).

CEPAL/UNESCO, Educación y conocimiento: eje de la transformación productiva con equidad, LC/G.1702/Rev.2-P, serie libros de la CEPAL, No 33, Santiago de Chile (1992).

CIDE, Centro de Investigación y Desarrollo de la Educación, Octava encuesta Nacional de Actores del sistema educativo, Universidad Alberto Hurtado, (en línea), 2010, http://www.cide.cl/mailing/textos_completos/VIII_encuesta_web.pdf. Acceso: 26 de mayo (2011).

Cheng, W. y S. Wang, Applying a 3D virtual learning environment to facilitate student's application ability - The case of marketing, Computers and human behavior: 27, 576-584 (2011).

Cronin, P., Report on the application of virtual reality technology to education, University of Edinburgh (2000).

Dalgarno, B. y otros tres autores, Effectiveness of a virtual laboratory as a preparatory resource for distance education chemistry students, Computers and education: 53, 853-865 (2009).

De Lucia, A. y otros tres autores, Development and evaluation of a virtual campus on second life: the case of SecondDMI, Computers and education: 52, 220-233 (2009).

Díaz-Antón, G., Uso de software educativo de calidad como herramientas de apoyo para el aprendizaje, Jornadas educativas: la escuela como instrumento de cambio, IEA, Caracas (en línea), 2002. http://www.academia-interactiva/articulos.html. Acceso: 14 de marzo (2011). 
Escobar, A., Bienvenidos a Cyberia, notas para una antropología de la cibercultura, Revista de estudios sociales: 22, 15-35 (2005).

Fernández, R., Modelo Informático para la autogestión del aprendizaje para la universalización de la enseñanza, tesis de doctorado, Departamento de Ciencias Sociales y Humanas, Universidad de Granada, Granada, España (2005).

Fundación Omar Dengo., Educación y Tecnologías digitales, como valorar su impacto social y sus contribuciones a la equidad. San José, Costa Rica. Fundación Omar Dengo. 112 p (2006).

Galanxhi, H. y Nah, F., Deception in cyber- space: a comparison of text-only vs. Avatarsupported medium, International Journal of Human-Computer Studies: 65, 770-783 (2007).

Galvis, A., Ingeniería de software educativo. Uniandes (1992).

García, M. y Benítez, A., Competencias Matemáticas Desarrolladas en Ambientes Virtuales de Aprendizaje: el Caso de MOODLE. Formación Universitaria, ISSN: 0718-5006 (en línea), 4 (3), 2011. http://www.scielo.cl/scielo.php?script=sci_arttext\&pid=S0718-

50062011000300005\&lng=es\&nrm=iso. Acceso: 1 de Noviembre (2011)

Gibson, W., Neuromancer, $1^{\mathrm{a}}$ edición, 1-252. Minotauro, Barcelona, España (1996).

Gros, B., Juegos digitales para comprender sistemas complejos, Revista Comunicación y Pedagogía (en línea), 2006, http://www.xtec.es/ abernat/articles/gros-II.pdf. Acceso: 25 de marzo (2011).

Grüsser, S.M., R. Thalemann, y M.D. Griffiths, Results of this study contribute to the assumption that also playing games without monetary reward meets criteria of addiction, Cyberpsychology and behavior: 10(2), 209-292 (2007).

Guri-Rosenblit, S., Distance and campus universities: Tensions and interactions - A comparative study of five countries, Oxford: IAU Press Pergamon (1999).

Hamada, M., An example of virtual reality and web-based application in learning, The International Journal of Virtual Reality: 7(3), 1-8 (2008).

Helsel, S., Virtual reality and education, Educational Technology: 32(5), 38-42 (1992).

Hernández, G. y V. Romero, Posibilidades de uso de las TIC en prácticas educativas universitarias mediante la modalidad b-learning, primer congreso latinoamericano de ciencias de la educación, 1-12, Baja California, México 27 a 27 de Septiembre (2010).

Hopenhayn, M., Educación, Comunicación y Cultura en la sociedad de la información: una perspectiva latinoamericana, Revista de la CEPAL: 81, 175-193 (2003).

Hrastinski, S., Introducing an informal synchronous medium in a distance learning course: How is participation affected? The Internet and Higher Education: 9, 117-131 (2006).

Hrastinski, S., Participating in synchronous online education, Ph.D. Thesis, Department of Informatics, Lund University, Sweden (2007).

Kontogeorgiou, A.M., J. Bellou y A.T. Mikropoulos, Being inside the quantum atom, PsychNology Journal: 6(1), 83-98 (2008).

Kreijns, K., P. Kirschner y W. Jochems, Identifying the pitfalls for social interaction in computersupported collaborative learning environments: A review of the research, Computers and Human Behavior: 19, 335-353 (2003). 
Lévy, P., Cibercultura: la cultura de la sociedad digital, Antrophos, México (2007).

López, Y., Diseño de un Programa Computacional Educativo (Software) para la Enseñanza de Balance General. Rev. Formación Universitaria, ISSN: 0718-5006 (en línea), 4(3), 2011. http://www.scielo.cl/scielo.php?script=sci_arttext\&pid=S0718-

50062011000300004\&lng=es\&nrm=iso. Acceso: 1 de Noviembre (2011)

Marquès, G.P., Impacto de las TIC en educación: funciones y limitaciones (en línea), 2008, http://dewey.uab.es/PMARQUES/siyedu.htm. Acceso: 24 de marzo (2011).

Mikropoulos, T.A. y A. Natsis, Educational virtual environments: A ten - year review of empirical research (1999 - 2009), Computers and education: 56, 769-780 (2011).

Mikropoulos, T.A. y V. Strouboulis, Factors that influence presence in educational virtual environments, CyberPsychology and Behavior: 7(5), 582-591 (2004).

Ministerio de Educación, Implementación curricular en educación media técnico profesional (en línea), 2009, http://www.formaciontecnica.cl/noticias/10-Seminario-de-difusi\%F3n-de-Estudios-deFormaci\%F3n-T\%E9cnico-Profesional.html. Acceso: 25 de abril (2011).

Palloff, R. y Pratt, K., Building learning communities in cyberspace - Effective strategies for the online classroom, San Fransisco: Jossey-Bass Publishers (1999).

Pan, Z. y otros cuatro autores, Virtual reality and mixed reality for virtual learning environments, Computers and graphics: 30, 20-28 (2006).

Pantelidis, V., Virtual reality in the classroom, Educational Technology: 33(4), 23-27 (1993).

Prensky, M., Digital Natives, Digital Inmigrants. On the horizont 9, (en línea), 2001, http://www.marcprensky.com/writing/Prensky\%20\%20Digital\%20Natives,\%20Digital\%20Immigrant s\%20-\%20Part1.pdf. Acceso: 25 de marzo (2011).

Proto, C., Profesores innovadores: sus prácticas, estilos y creencias, Educar Chile: 1-22 (2010).

Quinche, J. y González, F., Entornos Virtuales 3D, Alternativa Pedagógica para el Fomento del Aprendizaje Colaborativo y Gestión del Conocimiento en Uniminuto. Rev. Formación Universitaria, ISSN: 0718-5006 (en línea), 4(2), 2011.

http://www.scielo.cl/scielo.php?script=sci_arttext\&pid=S0718-

50062011000200006\&lng=es\&nrm=iso. Acceso: 1 de Noviembre (2011)

Rosas, R. y otros once autores, Beyond Nintendo: design and assessment of educational video game for first and second grade students, Computer and Education: 40(1) (2003).

Rourke, L. y otros tres autores, Assessing social presence in asynchronous text-based computer conferencing, Journal of Distance Education: 14(2), 50-71 (2001).

Salzman, M, y otros tres autores, A Model for Understanding How Virtual Reality Aids Complex Conceptual Learning, Presence: Teleoperators and Virtual Environments: 8(3), 293-316 (1999)

Selwin, N., The use of computer technology in university teaching and learning: a critical perspective, Journal of computer assisted learning: 23, 83-94 (2007).

Serrano, A. y E. Martínez, La Brecha Digital: mitos y realidades, $1^{\mathrm{a}}$ edición, 1-125, Editorial Universitaria UABC, Baja California, México (2003).

Smith - Robbins, S., "This game sucks": how to improve the gamification of education, New horizonts: the technologies ahead, Educause review: 58-59 (2011). 
Sosa, R. y otros, B-learning y teoría del aprendizaje constructivista, disciplinas informáticas: un esquema de ejemplo a aplicar, Recent research developments in learning Technologies (en línea), 2005, http://www.formatex.org/micte2005/AprendizajeConstructivista.pdf. Acceso: 25 de marzo (2011).

Traphagan, T.W. y otros nueve autores, Cognitive, social and teaching presence in a virtual World and a text chat, Computers and education: 55, 923-936 (2010).

UNESCO, ICTS for Higher Education (en línea), 2009,

http://unesdoc.unesco.org/images/0018/001832/183207e.pdf. Acceso: 1 de Noviembre (2011)

Vallverdú, J., Apuntes epistemológicos a la e-ciencia, Revista de Filosofía: 64, 193-214 (2008).

Vasalou, A. y Joinson A.M., Me, myself and $\mathrm{l}$ : the role of interactional context on self-presentation through avatars, Computers in human behavior: 25, 510-520 (2009).

Winn, W. y M. Windschitl, Learning science in virtual environments: the interplay of theory and experience, Themes in Education: 1(4), 373-389 (2000).

Winn, W., A conceptual basis for educational applications of virtual reality (Report No. TR-93-9). Human Interface Technology Laboratory, Washington Technology Center (1993). 